\title{
ESTIMATES FOR DERIVATIVES OF RATIONAL FUNCTIONS AND THE FOURTH ZOLOTAREV PROBLEM
}

\author{
A. L. LUKASHOV
}

\begin{abstract}
An estimate is obtained for the derivatives of real rational functions that map a compact set on the real line to another set of the same kind. Many well-known inequalities (due to Bernstein, Bernstein-Szegő, V. S. Videnskiı̌, V. N. Rusak, and M. Baran-V. Totik) are particular cases of this estimate. It is shown that the estimate is sharp. With the help of the solution of the fourth Zolotarev problem, a class of examples is constructed in which the estimates obtained turn into identities.
\end{abstract}

\section{$\S 1$. INTRODUCTION}

The Bernstein-Markov inequalitites for derivatives of trigonometric and algebraic polynomials, as well as their various generalizations, play an important role in approximation theory (see, e.g., the books $[1,2,3]$ and also the recent papers $[4,5,6,7]$ and the references therein).

Our goal in the present paper is to obtain an inequality estimating the derivative of a real rational function that maps a given compact set $E \subset \mathbb{R}$ to another compact set $F \subset \mathbb{R}$. We show that this inequality is sharp for any $F$ and that it turns into the identity for a wide class of rational functions in the case where $F$ is the union of two segments.

We consider rational functions of degree $n$ of the form

$$
R_{n}(x)=\frac{P_{n}(x)}{Q_{n}(x)}
$$

where $P_{n}$ and $Q_{n}$ are real polynomials of degree at most $n$. Let $x_{1}, \ldots, x_{n}$ be the zeros of $Q_{n}$ (if $\operatorname{deg} Q_{n}=n-k<n$, we assume that $x_{n-k+1}=\cdots=x_{n}=\infty$ ). We shall use the harmonic measure $\omega(z, e, \Omega)$ of a set $e \subset E=\partial \Omega$ at a point $z$ relative to a domain $\Omega=\mathbb{C} \backslash E$, and also the corresponding density $\varpi_{E}(z, x)=\frac{d}{d x}(\omega(z,[\inf E, x] \cap E, \Omega))$. Next, we denote by $Z_{n}(x)$ the solution of the fourth Zolotarev problem (see [8, 9, 10]) concerning the best approximation of the function $\operatorname{sgn} x$ on $E=[-1, \kappa] \cup[\kappa, 1], 0<\kappa<1$, by real rational functions of degree at most $n$.

We cite one of the main results of [7].

\section{Theorem 1. Let}

$$
r(x)=\frac{x^{n}+b_{1} x^{n-1}+\cdots+b_{n}}{\sqrt{\rho_{\nu}(x)}},
$$

where $b_{1}, \ldots, b_{n} \in \mathbb{R}$, and $\rho_{\nu}(x)=c \prod_{j=1}^{\nu^{*}}\left(x-x_{j}\right)^{\nu_{j}}$ is a real polynomial of degree $\nu$ that is positive on $E=\left[a_{1}, a_{2}\right] \cup \cdots \cup\left[a_{2 l-1}, a_{2 l}\right] \subset \mathbb{R}, a_{1}<\cdots<a_{2 l}$. Then for any $x \in \operatorname{int} E$

2000 Mathematics Subject Classification. Primary 53A04; Secondary 52A40, 52A10.

Key words and phrases. Estimates of derivatives, optimal filter, Zolotarev problems.

Supported by RFBR (grant no. 07-01-00167). 
we have

$$
\left(\frac{r^{\prime}(x)}{\frac{\pi}{2}\left((2 n-\nu)_{+} \varpi_{E}(\infty, x)+\sum_{j=1}^{\nu^{*}} \nu_{j} \varpi_{E}\left(x_{j}, x\right)\right)}\right)^{2}+r^{2}(x) \leq\|r\|_{C(E)}^{2} ;
$$

moreover, equality occurs for all $x \in \operatorname{int} E$ whenever the following condition on the set $E$ is fulfilled:

$$
\left(n-\frac{\nu}{2}\right) \omega\left(\infty,\left[a_{2 j-1}, a_{2 j}\right], \mathbb{C} \backslash E\right)+\frac{1}{2} \sum_{k=1}^{\nu^{*}} \nu_{k} \omega\left(x_{k},\left[a_{2 j-1}, a_{2 j}\right], \mathbb{C} \backslash E\right)=q_{j}, \quad q_{j} \in \mathbb{N},
$$

$j=1, \ldots, l$, and the function $r(x)$ admits the representation

$$
r(x)=\text { const } \cos \left(\pi \int_{a_{1}}^{x}\left(\left(n-\frac{\nu}{2}\right) \varpi_{E}(\infty, x)+\frac{1}{2} \sum_{j=1}^{\nu^{*}} \nu_{j} \varpi_{E}\left(x_{j}, x\right)\right) d x\right) .
$$

This theorem was presented in [7] without proof (and was formulated without the equality case), but its justification practically repeats the proof of [7, Theorem 4]. The only modification is that, for replacement of a "zero" at infinity, the polynomial in question should be multiplied by a factor of the form $\frac{1}{M^{2}}\left(x^{2}+M^{2}\right)$, which tends to 1 uniformly on $E$ as $M \rightarrow \infty$ (instead of the trigonometric factor $\frac{4}{e^{M}} \sin \frac{\varphi-M i}{2} \sin \frac{\varphi+M i}{2}$ ).

Our main result in the present paper is as follows.

Theorem 2. If a function $R_{n}$ of the form (1) maps a compact set $E \subset \mathbb{R}$ to a compact set $F \subset \mathbb{R}$, then for any inner point $x$ in $E$ we have

$$
\left|R_{n}^{\prime}(x)\right| \leq \frac{\sum_{k=1}^{n} \varpi_{E}\left(x_{k}, x\right)}{\varpi_{F}\left(\infty, R_{n}(x)\right)} .
$$

Since $\varpi_{[-1,1]}(\infty, y)=\frac{1}{\pi \sqrt{1-y^{2}}}$, Theorem 2 implies Theorem 1 with $\rho_{\nu}=Q_{n}^{2}(x)$ in the case where $F=[-1,1], E=\left[a_{1}, a_{2}\right] \cup \cdots \cup\left[a_{2 l-1}, a_{2 l}\right]$. In its turn, this yields a series of known inequalities (a more detailed account of the history of this topic can be found in $[7])$.

(1) Suppose $F=[-1,1], E=[-1,1]$, and $\rho_{\nu}(x) \equiv 1$; we obtain the Bernstein-Szegő inequality

$$
\left|P_{n}^{\prime}(x)\right| \leq \frac{n \sqrt{1-P_{n}^{2}(x)}}{\sqrt{1-x^{2}}}
$$

valid for any $x \in(-1,1)$ and any polynomial $P_{n}(x)$ of degree at most $n$, and, as a consequence, the classical Bernstein inequality

$$
\left|P_{n}^{\prime}(x)\right| \sqrt{1-x^{2}} \leq n\left\|P_{n}\right\|_{C([-1,1])} .
$$

(2) Suppose $F=[-1,1], E=[-1,1]$, and $\rho_{\nu}(x)$ is an arbitrary real polynomial; we obtain Rusak's inequality

$$
\left|r_{n}^{\prime}(x)\right| \leq \sqrt{1-r_{n}^{2}(x)} \frac{\left|\lambda_{n}(x)\right|}{\sqrt{1-x^{2}}}, \quad x \in(-1,1),
$$

where $r_{n}(x)=\frac{p_{n}(x)}{\sqrt{\prod_{k=1}^{2 n}\left(1+a_{k} x\right)}}$, the $a_{k}$ are either reals with $\left|a_{k}\right|<1$ or pairwise mutually conjugate complex numbers, $p_{n}$ is an algebraic polynomial of degree at most $n$ with real coefficients, and $\lambda_{n}(x)=\frac{1}{2} \sum_{k=1}^{2 n} \frac{\sqrt{1-a_{k}^{2}}}{1+a_{k} x}$. As a consequence, we also get Videnskii's inequality

$$
\left|r_{n}^{\prime}(x)\right| \sqrt{1-x^{2}} \leq\left|\lambda_{n}(x)\right|
$$

which is valid now for any complex $p_{n}(x)$. 
(3) Suppose $F=[-1,1], E$ is an arbitrary compact subset of $\mathbb{R}$, and $\rho_{\nu}(x) \equiv 1$; we arrive at the inequality

$$
\left(\frac{\left|P_{n}^{\prime}(x)\right|}{\pi \omega_{E}(x)}\right)^{2}+n^{2} P_{n}^{2}(x) \leq n^{2}\left\|P_{n}\right\|_{C(E)}^{2}, \quad x \in E,
$$

which is due to Baran and Totik. Here $P_{n}$ is an arbitrary real algebraic polynomial of degree at most $n$, and $\omega_{E}(x)=\varpi_{E}(\infty, x)$. We mention that Baran obtained even a multidimensional generalization of (7). Inequality (7) leads to the Bernstein type estimate established by Akhiezer and Levin for the more general case of entire functions of exponential type.

Now we present results that show the sharpness of our inequality.

Theorem 3. Let $T_{N}(x)$ be a real polynomial of degree $N$ all zeros of which are real and simple, and let $A=\min _{x: T_{N}^{\prime}(x)=0}\left|T_{N}(x)\right|$. Let $\alpha, \beta, \gamma, \delta$ be real numbers satisfying $-A<\alpha<\beta<\gamma<\delta<A, \beta-\alpha=\delta-\gamma$. Take $[\alpha, \beta] \cup[\gamma, \delta]$ for the role of $F$ and the inverse image $T_{N}^{-1}(F)$ for the role of $E$. Then for any $n \in \mathbb{N}$ there exists a rational function $R_{n N}(x)$ of degree $n N$ such that for all inner paints of $E$ we have

$$
\left|R_{n N}^{\prime}(x)\right|=\frac{\sum_{k=1}^{n N} \varpi_{E}\left(x_{k}, x\right)}{\varpi_{F}\left(\infty, R_{n N}(x)\right)},
$$

where $x_{1}, \ldots, x_{n N}$ are the poles of $R_{n N}(x)$ (some of them may be equal to $\infty$ if the degree of the denominator is smaller than that of the numerator).

Corollary 1. Let $Z_{n}(x)$ be the solution of the fourth Zolotarev problem (on the best approximation of the function $\operatorname{sgn} x$ on $E=[-1,-\kappa] \cup[\kappa, 1], 0<\kappa<1$, by real rational functions), and let $F=Z_{n}(E)$. Then for all $x \in \operatorname{int} E$ we have

$$
\left|Z_{n}^{\prime}(x)\right|=\frac{\sum_{k=1}^{n} \varpi_{E}\left(x_{k}, x\right)}{\varpi_{F}\left(\infty, Z_{n}(x)\right)}
$$

where the $x_{k}$ are the poles of $Z_{n}(x)$, and $x_{n}=\infty$ for $n$ odd.

Theorem 4. For any compact set $F$, any $\varepsilon>0$, any $n \in \mathbb{N}$, any $y_{0} \in \operatorname{int} F$, and any real or pairwise complex conjugate poles $x_{1}, \ldots, x_{n} \in \overline{\mathbb{C}}$, there exists a rational function $R_{n}$ of degree $n$ with poles $x_{1}, \ldots, x_{n}$ and a compact set $E$ such that for some point $x_{0} \in E$ we have $R_{n}\left(x_{0}\right)=y_{0}, R_{n}(E) \subset F$, and

$$
\left|R_{n}^{\prime}\left(x_{0}\right)\right|>\frac{\sum_{k=1}^{n} \varpi_{E}\left(x_{k}, x_{0}\right)}{\varpi_{F}\left(\infty, y_{0}\right)}(1-\varepsilon) .
$$

\section{$\S 2$. Proofs}

Proof of Theorem 2. First, let $E$ and $F$ be finite unions of segments in $\mathbb{R}$. If $F$ consists of one segment, then the claim of Theorem 2 coincides with Theorem 1, because

$$
\omega\left(\infty, e, \mathbb{C} \backslash\left[-\left\|R_{n}\right\|_{C(E)},\left\|R_{n}\right\|_{C(E)}\right]\right)=\frac{1}{\pi} \int_{e} \frac{1}{\sqrt{\left\|R_{n}\right\|_{C(E)}^{2}-y^{2}}} d y .
$$

Now, suppose that $F$ consists of more than one segment. Arguing by contradiction, we assume that there exists a rational function of the form (1) that maps the system of segments $E$ to the system of segments $F$ and satisfies

$$
\left|R_{n}^{\prime}\left(x_{0}\right)\right|>\frac{\sum_{k=1}^{n} \varpi_{E}\left(x_{k}, x_{0}\right)}{\varpi_{F}\left(\infty, R_{n}\left(x_{0}\right)\right)}
$$

for some $x_{0} \in \operatorname{int} E$. By an arbitrarily small perturbation of the endpoints of the segments forming the system $F$, we can turn $F$ into a system $\tilde{F}$ having the same number of 
segments, but such that the harmonic measure of each of these new segments is rational (this fact was implicit in [11] and was proved in [13, 12, 14, 15]). Consequently, we may assume that inequality $(9)$ is valid also for the new system $\tilde{F}$. The system of segments $\tilde{F}$ is the preimage of $[-1,1]$ under some polynomial map $T_{N}$, i.e., $\tilde{F}=T_{N}^{-1}([-1,1])$, $\operatorname{deg} T_{N}=N$ (see $\left.[16,17,15]\right)$. Consider the function $R_{n N}(x):=T_{N}\left(R_{n}(x)\right)$, which is a rational function of degree $n N$ and has the form $T_{N}\left(R_{n}(x)\right)=P_{n N}(x) / Q_{n}^{N}(x)$, where $P_{n N}(x)$ is a polynomial of degree at most $n N$. We calculate the derivative

$$
R_{n N}^{\prime}(x)=T_{N}^{\prime}\left(R_{n}(x)\right) R_{n}^{\prime}(x) .
$$

Since $T_{N}(y)$ satisfies the differential equation

$$
\frac{\left(T_{N}^{\prime}(y)\right)^{2}}{\left(\pi N \varpi_{\tilde{F}}(\infty, y)\right)^{2}}+T_{N}^{2}(y)=1
$$

(see [18] or Theorem 1), we see that

$$
\left|R_{n N}^{\prime}\left(x_{0}\right)\right|=\sqrt{1-T_{N}^{2}\left(R_{n}\left(x_{0}\right)\right)} \pi N \varpi_{\tilde{F}}\left(\infty, T_{N}\left(x_{0}\right)\right)\left|R_{n}^{\prime}\left(x_{0}\right)\right| .
$$

Using inequality (9), we obtain

$$
\left|R_{n N}^{\prime}\left(x_{0}\right)\right|>\pi N \sqrt{1-T_{N}^{2}\left(R_{n}\left(x_{0}\right)\right)} \sum_{k=1}^{n} \varpi_{E}\left(x_{k}, x_{0}\right),
$$

which contradicts Theorem 1 . Of course, we should remember that $R_{n}\left(x_{0}\right)$ cannot be a boundary point of the system of segments $\tilde{F}$, because otherwise $x_{0}$ would be a point of local extremum for $R_{n}$, so that the derivative $R_{n}^{\prime}$ would be equal to zero at $x_{0}$, in contradiction with (9). Passage from the system of segments $E$ to an arbitrary compact set can be done in the same way as in [15, Theorem 3.2]. The same passage for the set $F$ is similar. Theorem 2 is proved.

Proof of Theorem 3. By the choice of $\alpha, \beta, \gamma, \delta$, the set $E$ consists of $2 N$ segments such that $N$ of them are mapped by the polynomial $T_{N}$ onto $[\alpha, \beta]$, and the other $N$ segments are mapped onto $[\gamma, \delta]$. Next, let $z=Z_{n}(x)=P_{n}(x) / Q_{n}(x)$ be the solution of the fourth Zolotarev problem (see Corollary 1). It is known (see [19, Appendices, Subsection 35]) that if $n$ is even, then $\operatorname{deg} P_{n}=n-1, \operatorname{deg} Q_{n}=n$, and if $n$ is odd, then $\operatorname{deg} P_{n}=n$, $\operatorname{deg} Q_{n}=n-1$.

Also, on the segment $[\kappa, 1]$ the values of $z$ run through the segment $[1-\mathcal{L}, 1+\mathcal{L}]$, taking alternately the values $1-\mathcal{L}$ and $1+\mathcal{L}$ at $n+1$ points, and on $[-1,-\kappa]$ the values of $z$ run through $[-1-\mathcal{L},-1+\mathcal{L}]$, taking alternately the values $-1-\mathcal{L}$ and $-1+\mathcal{L}$ at $n+1$ points; here $\mathcal{L}$ is the size of the deviation. Then the function

$$
R_{n N}(x):=Z_{n}\left(\frac{T_{N}(x)-\frac{\beta+\gamma}{2}}{\delta-\frac{\beta+\gamma}{2}}\right)
$$

maps $E$ onto $[-1-\mathcal{L},-1+\mathcal{L}] \cup[1-\mathcal{L}, 1+\mathcal{L}]$, and on each segment $\Delta$ of $E$ it takes alternately either the values $1-\mathcal{L}, 1+\mathcal{L}\left(\right.$ if $\left.T_{N}(\Delta)=[\gamma, \delta]\right)$, or the values $-1-\mathcal{L},-1+\mathcal{L}$ (if $T_{N}(\Delta)=[\alpha, \beta]$ ). Consequently, the function

$$
\frac{1}{2 \mathcal{L}} R_{n N}^{2}(x)-\frac{1+\mathcal{L}^{2}}{2 \mathcal{L}}
$$

maps $E$ onto $[-1,1]$, and it has the maximal number $(2(n+1) N=2 n N+2 N)$ of points of deviation, i.e., points where it equals \pm 1 . Then this function satisfies the identity (see 
Theorem 1)

$$
\left|\left(\frac{1}{2 \mathcal{L}} R_{n N}^{2}(x)-\frac{1+\mathcal{L}^{2}}{2 \mathcal{L}}\right)^{\prime}(x)\right|=2 \pi \sum_{k=1}^{n N} \varpi_{E}\left(x_{k}, x\right) \sqrt{1-\left(\frac{1}{2 \mathcal{L}} R_{n N}^{2}(x)-\frac{1+\mathcal{L}^{2}}{2 \mathcal{L}}\right)^{2}}
$$

at every inner point $x$ of $E$; here the $x_{k}$ are the same as in the statement of the theorem. This implies that

$$
\left|y^{\prime}(x)\right|=\frac{1}{\left|\frac{y(x)}{\pi \sqrt{4 y^{2}(x)-\left(y^{2}(x)-\mathcal{L}^{2}+1\right)^{2}}}\right|} \sum_{k=1}^{n N} \varpi_{E}\left(x_{k}, x\right)
$$

for $x \in \operatorname{int} E$, where $y=R_{n N}(x)$. To complete the proof of Theorem 3, it remains to observe that the harmonic measure density $\varpi_{F}(\infty, y)$, i.e., the density of the equilibrium measure for $[-1-\mathcal{L},-1+\mathcal{L}] \cup[1-\mathcal{L}, 1+\mathcal{L}]$, is equal to

$$
\frac{y}{\pi \sqrt{-\left((y-1)^{2}-\mathcal{L}^{2}\right)\left((y+1)^{2}-\mathcal{L}^{2}\right)}}
$$

(see $[21,20])$, which coincides with the denominator on the right-hand side in (10).

To verify the corollary, it suffices to put $T_{n}(x)=x$ in the above proof of Theorem 3 ; then $A=\infty$ and $\alpha=-1, \beta=-\kappa, \gamma=\kappa, \delta=1$.

Proof of Theorem 4. We choose $F^{*}=T_{N}^{-1}([-1,1])$ so that $F \subset F^{*}$ and

$$
\varpi_{F^{*}}\left(\infty, y_{0}\right)<\varpi_{F}\left(\infty, y_{0}\right) \frac{1}{1-\varepsilon}
$$

(see [15]), and

$$
\left|T_{N}\left(y_{0}\right)\right|<1 .
$$

Now, we take an arbitrary real rational function of degree $n$ that has $n$ real simple zeros and the given poles $x_{1}, \ldots, x_{n}$. Multiplying it by a sufficiently large constant, we may assume that for the resulting function $R_{n}$ we have $F^{*} \subset(-A, A)$, where $A=$ $\min _{x: R_{n}^{\prime}(x)=0}\left|R_{n}(x)\right|$. Let $E$ be the preimage of the set $F^{*}$ under the map $R_{n}$. If $F^{*}$ consists of $m$ segments, then $E$ consists of $m n$ segments. Let $x_{0}$ be any solution of the equation $R_{n}\left(x_{0}\right)=y_{0}$. Since for all $\alpha \in[-1,1]$ all roots of the equation $T_{N}\left(R_{n}(x)\right)-\alpha=0$ lie in the system $E$ of segments, we see that $T_{N}\left(R_{n}(x)\right)$ is a rational function that realizes the smallest deviation from zero on $E$ and has the maximal number of deviation points. Therefore, this function satisfies the identity

$$
\left|\left(T_{N}\left(R_{n}(x)\right)\right)^{\prime}\right|=N \pi \sum_{k=1}^{n} \varpi_{E}\left(x_{k}, x\right) \sqrt{1-T_{N}^{2}\left(R_{n}(x)\right)}
$$

for all $x \in \operatorname{int} E$. Similarly (see also $[18,15]$ ), for $T_{N}(y)$ we have

$$
\left|T_{N}^{\prime}(y)\right|=N \pi \varpi_{F^{*}}(\infty, y) \sqrt{1-T_{N}^{2}(y)}
$$

for all $y \in \operatorname{int} F^{*}$. Relations (12)-(14) imply that

$$
\left|R_{n}^{\prime}\left(x_{0}\right)\right|=\frac{\sum_{k=1}^{n} \varpi_{E}\left(x_{k}, x_{0}\right)}{\varpi_{F^{*}}\left(\infty, R_{n}\left(x_{0}\right)\right)} .
$$

It remains to use inequality (11).

The author thanks the referee for advice and remarks which contributed to improving the initial version of the paper.

Remark 1. Theorem 2 was announced in [22]. 
Remark 2. By using [7, Theorems 2 and 4], it is possible to prove a result similar to Theorem 2 for the ratio of trigonometric polynomials. This result was announced in [23].

Remark 3. The arguments employed in the reduction of the fourth Zolotarev problem to the third problem allow us to use the rational functions constructed in the proof of Theorem 3 for constructing sharp solutions with the maximal number of extremal points for the problem of synthesis of a many-band electric filter [24]. Such solutions, together with more general ones, were described in [25] (in terms of automorphic and elliptic functions).

\section{REFERENCES}

[1] P. Borwein and T. Erdélyi, Polynomials and polynomial inequalities, Grad. Texts in Math., vol. 161, Springer-Verlag, New York, 1995. MR1367960 (97e:41001)

[2] G. V. Milovanović, D. S. Mitrinović, and Th. M. Rassias, Topics in polynomials: Extremal problems, inequalities, zeros, World Sci. Publ. Co., Inc., River Edge, NJ, 1994. MR1298187 (95m:30009)

[3] Q. I. Rahman and G. Schmeisser, Les inégalités de Markoff et de Bernstein, Séminaire de Mathématiques Supérieures, vol. 86, Presses Univ. Montréal, Montreal, QC, 1983. MR0729316 (85f:41009)

[4] V. N. Dubinin, Conformal mappings and inequalities for algebraic polynomials, Algebra i Analiz 13 (2001), no. 5, 16-43; English transl., St. Petersburg Math. J. 13 (2002), no. 5, 717-738. MR1882862 (2003j:30010)

[5] T. Erdélyi and J. Szabados, On a generalization of the Bernstein-Markov inequality, Algebra i Analiz 14 (2002), no. 4, 36-53; English transl., St. Petersburg Math. J. 14 (2003), no. 4, 563-576. MR1935916 (2003k:41012)

[6] A. A. Pekarskiǔ, Bernstein-type inequalities for the derivatives of rational functions in $L_{p}$-spaces, $0<p<1$, on Lavrent'ev curves, Algebra i Analiz 16 (2004), no. 3, 143-170; English transl., St. Petersburg Math. J. 16 (2005), no. 3, 541-560. MR2083568 (2005f:30073)

[7] A. L. Lukashov, Inequalities for the derivatives of rational functions on several intervals, Izv. Ross. Akad. Nauk Ser. Mat. 68 (2004), no. 3, 115-138; English transl., Izv. Math. 68 (2004), no. 3, 543-565. MR2069196 (2006j:26010)

[8] E. I. Zolotarev, Application of elliptic functions to questions about functions with the lowest or the greatest deviation from zero, Collected Works. Vol. 2, Akad. Nauk SSSR, Leningrad, 1932, pp. 1-59. (Russian)

[9] N. I. Akhiezer, On an E. I. Zolotarev problem, Izv. Akad. Nauk SSSR Otdel. Fiz.-Mat. Nauk 1929, no. 10, 919-931. (Russian)

[10] A. A. Gonchar, The problems of E. I. Zolotarev which are connected with rational functions, Mat. Sb. (N.S.) 78 (1969), no. 4, 640-654; English transl., Math. USSR-Sb. 7 (1969), 623-635. MR0254238 (40:7447)

[11] R. M. Robinson, Conjugate algebraic integers in real point sets, Math. Z. 84 (1964), 415-427. MR0164956 (29:2247)

[12] H. P. McKean and P. van Moerbeke, Hill and Toda curves, Comm. Pure Appl. Math. 33 (1980), 23-42. MR0544043 (81b:14016)

[13] A. B. Bogatyrev, On the efficient computation of Chebyshev polynomials for several intervals, Mat. Sb. 190 (1999), no. 11, 15-50; English transl., Sb. Math. 190 (1999), no. 11-12, 1571-1605. MR1735137 (2001j:65030)

[14] F. Peherstorfer, Deformation of minimal polynomials and approximation of several intervals by an inverse polynomial mapping, J. Approx. Theory 111 (2001), 180-195. MR1849545 (2002g:41009)

[15] V. Totik, Polynomial inverse images and polynomial inequalities, Acta Math. 187 (2001), 139-160. MR1864632 (2002h:41017)

[16] A. I. Aptekarev, Asymptotic properties of polynomials orthogonal on a system of contours, and periodic motions of Toda chains, Mat. Sb. (N.S.) 125 (1984), no. 2, 231-258; English transl., Math. USSR-Sb. 53 (1986), no. 1, 233-260. MR0764479 (86g:35166)

[17] F. Peherstorfer, Orthogonal and extremal polynomials on several intervals, J. Comput. Appl. Math. 48 (1993), 187-205. MR 1246858 (94m:42058)

[18] _ On Bernstein-Szegő orthogonal polynomials on several intervals. II. Orthogonal polynomials with periodic recurrence coefficients, J. Approx. Theory 64 (1991), 123-161. MR1091466 (92a:42031) 
[19] N. I. Akhiezer, Lectures in the theory of approximation, 2nd ed., "Nauka", Moscow, 1965; English transl. of 1st ed., Frederick Ungar Publ. Co., New York, 1956. MR0188672 (32:6108); MR0095369 $(20: 1872)$

[20] H. Widom, Extremal polynomials associated with a system of curves in the complex plane, Adv. Math. 3 (1969), 127-232. MR0239059 (39:418)

[21] E. B. Saff and V. Totik, Logarithmic potentials with external fields, Grundlehren Math. Wiss., Bd. 316, Springer-Verlag, Berlin, 1997. MR1485778 (99h:31001)

[22] A. L. Lukashov, Inequalities for derivatives of rational functions, Complex Analysis and Its Applications, Abstracts of Reports, Krasnodar, 2005, pp. 70-71. (Russian)

[23] _ Inequalities for derivatives of trigonometric rational functions, Current Problems of Mathematics, Mechanics, and Informatics, Abstracts of Reports, Tula, 2005, pp. 116-117. (Russian)

[24] V. N. Malozemov, The synthesis problem for a multipole electrical filter, Zh. Vychisl. Mat. i Mat. Fiz. 19 (1979), no. 3, 601-609. (Russian) MR0538929 (80g:94005)

[25] A. L. Lukashov, A precise solution of the problem of constructing an optimal electric filter, Investigations in Algebra, Number Theory, Functional Analysis, and Related Topics, Vyp. 1, Saratov, 2003, pp. 84-90. (Russian)

N. G. Chernyshevskĭ Saratov State University, Astrakhanskaya 83, 410012, Saratov, RusSIA

Current address: Department of Mathematics, Fatih University, 34900 Büyükçekmece, Istanbul, Turkey

E-mail address: alexeylukashov@yahoo.de

Received 11/OCT/2006

Translated by A. PLOTKIN 